\title{
The thermoelastic response of cracked polycrystals with hexagonal symmetry
}

\author{
F. Willot ${ }^{\mathrm{a}, \mathrm{b}^{*}}$, H. Trumel ${ }^{\mathrm{c}}$ and D. Jeulin ${ }^{\mathrm{a}, \mathrm{b}}$ \\ ${ }^{a}$ Mines ParisTech, PSL - Research University, Centre for Mathematical Morphology, 35 rue Saint-Honoré, \\ 77300 Fontainebleau, France; b Mines ParisTech, PSL - Research University, Centre for Materials, 63-65 Rue \\ Henri Auguste Desbruères, 91100 Corbeil-Essonnes, France; ${ }^{c}$ CEA, DAM, Le Ripault, F-37260 Monts, France. \\ *Corresponding author. Email: francois.willot@ensmp.fr.
}

\section{REFERENCE}

Philosophical Magazine 99 (5), 606-630 (2019). DOI: 10.1080/14786435.2018.1547432.

\begin{abstract}
The influence of a population of randomly-oriented cracks on the macroscopic thermal and linearelastic response of a hexagonal polycrystal is addressed using a self-consistent method. Coupling between micro-cracks and crystal anisotropy is taken into account through the effective medium where all inhomogeneities are embedded. In the absence of cracks, the proposed approach reduces to the self-consistent estimate of Berryman (2005). The accuracy of the present method is first assessed using numerical, Fourier-based computations. In the absence of crystal anisotropy, the estimates for the effective elastic properties are close to that obtained numerically for a homogeneous body containing disk-shaped cracks, with Boolean spatial dispersion. Various other analytical estimates and bounds, that are available for homogeneous cracked bodies, are also considered and compared to the present approach. Second, the combined role of crystal anisotropy and micro-cracks is investigated analytically, specifically when the in-plane shear modulus of the crystal becomes zero. The cracks-density percolation threshold is found to diminish abruptly in this limit. This "advanced" percolation threshold is concomitant to the onset of large, weakly-loaded regions surrounding cracks in strongly-anisotropic crystals.
\end{abstract}

\section{KEYWORDS}

Cracked polycrystals; Homogenization; Thermoelasticity; Self-consistent Methods; Percolation

\section{Introduction}

Quasi-brittle materials represent a wide and important class of engineering materials, comprising concrete, soft rocks, sintered and jointed materials and many composites. Their common characteristic is to undergo distributed damage, namely micro-cracking, prior to failure. An important effect of microcracks is the induced decrease of the macroscopic elastic stiffness (elastic softening), whereas their effect on macroscopic thermal expansion does not necessarily follow the same trend. In the general case, elastic softening is accompanied by induced anisotropy, known to reflect the non-uniform distribution of micro-cracks orientations, itself the result of either the strong initial heterogeneity and anisotropy of the material, such as in fiber reinforced composites, or the directionality of the load, in initially isotropic materials such as concrete, un-textured rocks and many ceramics. From now on, the focus will be restricted to the latter case, and more precisely on initially isotropic polycrystals.

Modeling this softening effect is not straightforward, and has generated a large amount of work, either by phenomenological means (see in particular the brief review of Cormey and Welemane [1] or more recently [2-4]) or via micromechanical modeling (see [5-9], and the more recent studies [10-14] among many others). Most of these works have considered the damaged material as a homogeneous matrix containing a distribution of micro-cracks. However, real materials are not homogeneous in their initial state, and this assumption may be seen as being too restrictive, as noted for example by Ortiz [15], Pijaudier-Cabot and Bazant [16], or Challamel [4]. 
The problem however is potentially very complex, as it may involve open and closed cracks, complex unilateral effects due to strong local heterogeneities, closed crack kinking, and friction. Therefore, the present work will focus on a simplified problem, only involving open micro-cracks in a macroscopically isotropic material in its initial and damaged states as well, in a locally linear, thermoelastic framework. Initially isotropic materials should remain so if micro-cracking results from the application of a nondirectional load, in particular by heating or cooling slowly enough for the temperature field to remain uniform. Such a situation may be encountered in materials made of strongly thermally and elastically anisotropic crystals, such as polycrystalline graphite [17, 18], or the energetic material 1,3,5-triamino2,4,6-trinitrobenzene (TATB) $[19,20]$.

A previous attempt was made by Huang and $\mathrm{Hu}$ [21], aiming at predicting the elastic behavior of micro-cracked polycrystals, in the case of crystals of cubic symmetry. The present work is a first effort in making such predictions for other symmetries, in the present case hexagonal (transverse isotropy). This choice is motivated by applications to polycrystalline graphite or TATB, and because hexagonal symmetry is amenable to a relatively simple analytical treatment.

The proposed model uses the self-consistent approach. Exact expressions for the Hill tensor (or equivalently Eshelby's tensor) have been derived for cracks embedded in a transversely-isotropic [22] or orthotropic case [23], for particular crack shape and orientations. Closed-form explicit solutions of Eshelby's problem for an ellipsoidal crack in a medium with arbitrary anisotropy are not available. Therefore, in the present approach, we consider the grains, of any crystallographic orientation, and the randomly-oriented cracks as separate phases embedded in the homogeneous effective medium. Under this assumption, we do not take into account the exact interaction between an oriented crack and the crystallographic orientation of the surrounding medium.

The obtained formulation is first discussed in terms of its percolating behavior, and compared to previous models of microcracked materials. Quantitative model predictions are then confronted to numerical ones obtained by Fourier-based simulations, first on a homogeneous matrix in which (isotropic) populations of micro-cracks of various crack densities are embedded, then on polycrystalline microstructures representative of a TATB-based material, in which the same populations of micro-cracks as before are embedded. The confrontation uses the Bristow-Budiansky-O'Connell [5, 24] definition of microcrack density, widely accepted to be a good quantifier (see for example [3]), up to relatively large crack densities (up to around 0.7) representing several thousands of micro-cracks at most.

\section{Thermoelasticity problem for cracked polycrystals}

Consider a polycrystal in a domain $\Omega$ of volume $V$, containing a population of frictionless cracks along a set of surfaces $\Gamma$ and subjected to mechanical and thermal loading. In the rest of this work, it is assumed that the applied load results in the opening of all micro-cracks, so that:

$$
\boldsymbol{\sigma}(\boldsymbol{x}) \cdot \boldsymbol{n}=0, \quad \boldsymbol{x} \in \Gamma
$$

where $\boldsymbol{n}$ is the normal at point $\boldsymbol{x}$ along $\Gamma$ (oriented indiferently) and $\boldsymbol{\sigma}$ is the stress tensor. Outside of the cracks, the strain field $\varepsilon$ is determined as a function of the stress by the linear thermoelastic law:

$$
\varepsilon(\boldsymbol{x})=\mathbf{S}(\boldsymbol{x}): \boldsymbol{\sigma}(\boldsymbol{x})+\boldsymbol{\alpha}(\boldsymbol{x}) \Delta T, \quad \boldsymbol{\sigma}(\boldsymbol{x})=\mathbf{C}(\boldsymbol{x}):[\varepsilon(\boldsymbol{x})-\boldsymbol{\alpha}(\boldsymbol{x}) \Delta T], \quad \boldsymbol{x} \in \Omega \backslash \Gamma,
$$

where $\Delta T=T-T_{0}$ is the difference of temperature with respect to a reference temperature $T_{0}$. The permanent regime is attained at all time during cooling or heating, so that $\Delta T$ is homogeneous in $\Omega$ and represents a macroscopic thermal loading. Small deformation $\varepsilon_{i j}=(1 / 2)\left(\partial_{i} u_{j}+\partial_{j} u_{i}\right)$, with $\boldsymbol{u}$ the displacement vector, and quasi-static equilibrium, i.e. $\operatorname{div} \boldsymbol{\sigma} \equiv 0$ is assumed for all thermo-mechanical loadings. Hereafter, the compliance $\mathbf{S}$ and stiffness tensor $\mathbf{C}=\mathbf{S}^{-1}$ of the crystal in (2) have hexagonal (i.e. transversely-isotropic) symmetry. Equation (2) is rewritten as:

$$
\left(\varepsilon_{11}, \varepsilon_{22}, \varepsilon_{33}, 2 \varepsilon_{23}, 2 \varepsilon_{13}, 2 \varepsilon_{12}\right)^{\mathrm{t}}=\mathbf{S} \cdot\left(\sigma_{11}, \sigma_{22}, \sigma_{33}, \sigma_{23}, \sigma_{13}, \sigma_{12}\right)^{\mathrm{t}}+\boldsymbol{\alpha}^{\mathrm{t}} \Delta T
$$


where $\mathbf{S}$ is expressed, in the above Voigt notation, as the following $6 \times 6$ symmetric matrix:

$$
\mathbf{S}=\left(\begin{array}{cccccc}
1 / E_{p} & -\nu_{p} / E_{p} & -\nu_{p z} / E_{p} & 0 & 0 & 0 \\
& 1 / E_{p} & -\nu_{p z} / E_{p} & 0 & 0 & 0 \\
& & 1 / E_{z} & 0 & 0 & 0 \\
& & & 1 / G_{z p} & 0 & 0 \\
& & & & 1 / G_{z p} & 0 \\
& & & & & 2\left(1+\nu_{p}\right) / E_{p}
\end{array}\right)
$$

in an orthonormal basis $\left(e_{1}, e_{2}, e_{3}\right)$ local to each grain where $e_{3}$ coincides with the crystal's axis of symmetry. The compliance tensor $\mathbf{S}$ depends on five elastic moduli: the in-plane $\left(E_{p}\right)$ and out-of-plane $\left(E_{z}\right)$ Young moduli, the out-of-plane shear modulus $G_{z p}$, the in-plane $\left(\nu_{p}\right)$ and out-of-plane $\left(\nu_{p z}\right)$ Poisson ratios. In contrast to isotropic elasticity, Poisson effects depend on the loading direction, hence, the coefficients $\nu_{p}$ and $\nu_{p z}$ can exceed $1 / 2$. They vary in the range:

$$
-1 \leq \nu_{p} \leq 1-2 \nu_{p z}^{2} \frac{E_{z}}{E_{p}} \leq 1, \quad\left|\nu_{p z}\right| \leq \sqrt{\frac{\left(1-\nu_{p}\right) E_{p}}{2 E_{z}}} \leq \sqrt{\frac{E_{p}}{E_{z}}} .
$$

With the condition $E_{z} \geq 0, E_{p} \geq 0, G_{z p} \geq 0$, the above guarantees that $\mathbf{S}$ (or $\mathbf{C}$ ) is positive semidefinite. The two Young moduli $E_{z}$ and $E_{p}$ vary in the range:

$$
\frac{2 \nu_{z p}^{2}}{1-\nu_{p}} E_{p} \leq E_{z} \leq \frac{1-\nu_{p}}{2 \nu_{p z}^{2}} E_{p}, \quad \frac{2 \nu_{p z}^{2}}{1-\nu_{p}} E_{z} \leq E_{p} \leq E_{z} \frac{1-\nu_{p}}{2 \nu_{z p}^{2}}
$$

where we have introduced $\nu_{z p}=\nu_{p z} E_{z} / E_{p}$, inequalities (5) and (6) being equivalent. When needed, we refer to the following Voigt notation for stiffness tensors, equivalent to (3):

$$
\left(\sigma_{11}, \sigma_{22}, \sigma_{33}, \sigma_{23}, \sigma_{13}, \sigma_{12}\right)^{\mathrm{t}}=\mathbf{C} \cdot\left[\left(\varepsilon_{11}, \varepsilon_{22}, \varepsilon_{33}, 2 \varepsilon_{23}, 2 \varepsilon_{13}, 2 \varepsilon_{12}\right)^{\mathrm{t}}-\boldsymbol{\alpha}^{\mathrm{t}} \Delta T\right]
$$

where $\mathbf{C}$ is represented by the $6 \times 6$ matrix inverse of (4).

In the crystal, we assume that a purely-thermal loading induces transversely-isotropic dilation or contraction with the same axis of symmetry as that of $\mathbf{S}$. In the same basis used in (4), where $\mathrm{e}_{3}$ is the axis of symmetry of the crystal lattice, the thermal expansion tensor entering (3) reads, in vector notation:

$$
\boldsymbol{\alpha}(\boldsymbol{x})=\left(\alpha_{11}, \alpha_{11}, \alpha_{33}, 0,0,0\right)^{\mathrm{t}}
$$

i.e. $\alpha_{11}=\alpha_{22}$ and $\alpha_{12}=\alpha_{13}=\alpha_{23}=0$.

The polycrystal is a space-tessellation of $\Omega$, in which all grains are made from the same, randomlyoriented crystal. In an orthornormal basis $\left(x_{I}\right)$ attached to the laboratory:

$$
S_{I J, K L}(\boldsymbol{x})=R_{i I}(\boldsymbol{x}) R_{j J}(\boldsymbol{x}) R_{k K}(\boldsymbol{x}) R_{l L}(\boldsymbol{x}) S_{i j, k l}, \quad \alpha_{I J}(\boldsymbol{x})=R_{i I}(\boldsymbol{x}) R_{j J}(\boldsymbol{x}) \alpha_{i j}, \quad \boldsymbol{x} \in \Omega \backslash \Gamma,
$$

where $\mathbf{R}(\boldsymbol{x})$ are constant-per-grain rotation matrix and where $S_{i j, k l}$ and $\alpha_{i j}$ are given by (3), (4) and (8).

The effective thermoelastic response of the polycrystal reads:

$$
\overline{\boldsymbol{\sigma}}=\langle\boldsymbol{\sigma}(\boldsymbol{x})\rangle=\widetilde{\mathbf{C}}:(\overline{\boldsymbol{\varepsilon}}-\widetilde{\boldsymbol{\alpha}} \Delta T),
$$

where $\overline{\boldsymbol{\varepsilon}}=\langle\boldsymbol{\varepsilon}(\boldsymbol{x})\rangle$ and $\overline{\boldsymbol{\sigma}}$ are the macroscopic strain and stress fields, computed by averaging the fields $\boldsymbol{\varepsilon}(\boldsymbol{x})$ and $\boldsymbol{\sigma}(\boldsymbol{x})$ over the domain $\Omega$. In the following, the rotation matrix $\mathbf{R}$ and cracks's orientations are uniformly-distributed random variables in the space of 3D matrix rotations and in the sphere, respectively, and the polycrystal is statistically isotropic. In particular, the orientations of two different crystals are uncorrelated. 
The effective stiffness tensor $\widetilde{\mathbf{C}}$ reduces to its bulk modulus $\widetilde{K}$ and shear modulus $\widetilde{G}$ or equivalently, its Young modulus $\widetilde{E}$ and Poisson ratio $\widetilde{\nu}$, and the effective thermal expansion tensor is diagonal with the unique eigenvalue $\widetilde{\alpha}_{11}$. The macroscopic volumetric expansion is $\widetilde{\alpha}_{V}=\operatorname{tr}(\widetilde{\boldsymbol{\alpha}})=3 \widetilde{\alpha}_{11}$.

In the analytical treatment that follows, we use Eshelby-type solutions for the cracks, in a manner that model them as infinitesimally thin ellispoidal voids. Under this hypothesis, the strain field in the cracks is singular and each crack contributes to the spatial mean $\langle\varepsilon(\boldsymbol{x})\rangle$ by (see e.g. [9]):

$$
\frac{1}{V} \int_{\Gamma_{i}} \mathrm{~d} S \frac{\boldsymbol{b} \cdot \boldsymbol{n}+\boldsymbol{n} \cdot \boldsymbol{b}}{2},
$$

where $V$ is the volume of $\Omega, \Gamma_{i}$ the surface along crack $i$, dS an elementary surface along $\Gamma_{i}$ and $\boldsymbol{b}$ is the displacement jump at each point of $\Gamma_{i}$. In the above, denoting $\boldsymbol{u}^{ \pm}(\boldsymbol{x})$ the displacement vector field on each side of the lips of the cracks, $\boldsymbol{b}=\boldsymbol{u}^{+}-\boldsymbol{u}^{-}$where $\boldsymbol{n}$ is oriented positively from the - to the + side.

In a homogeneous body containing cracks, the effective elastic moduli depend on a non-dimensional cracks-density parameter $\eta$. This parameter arises naturally from Eshelby's single-inclusion problem in the "dilute limit" where cracks interaction are neglected [24] and has been subsequently used in most self-consistent schemes, e.g. [5]. When the cracks embedded in the polycrystal are equisized disks of radius $a$, the cracks-density parameter reads:

$$
\eta=\frac{n_{0} a^{3}}{V}
$$

where $n_{0}$ is the number of cracks in $\Omega$ and $n_{0} / V$ the number of cracks per unit volume. The cracks's locations are arbitrary, in particular, cracks may interpenetrate with one another. In the rest of this work, we assume that the spatial distribution of cracks in the polycrystal and their morphology can be described by the cracks-density parameter $0 \leq \eta \leq \infty$.

\section{Self-consistent estimate for cracked polycrystals}

We now derive estimates for the homogenized thermoelastic response of a cracked polycrystal using classical self-consistent assumptions. Elastic moduli are first considered. Our method is based on Eshelby's problem for: (i) an isolated penny-shaped crack in an isotropic medium and (ii) a spherical grain with transversely-isotropic elastic response embedded in an isotropic medium. Assume for now that the grains and cracks are isolated from one another and that they are embedded in a homogeneous isotropic body of compliance tensor $\mathbf{S}^{0}$, subjected to an applied stress $\overline{\boldsymbol{\sigma}}$. The contributions of the cracks and grains to the material's macroscopic strain $\bar{\varepsilon}$ are summed up as:

$$
\overline{\boldsymbol{\varepsilon}}=\widetilde{\mathbf{S}}: \overline{\boldsymbol{\sigma}}=\mathbf{S}^{0}: \overline{\boldsymbol{\sigma}}+f\left\langle\mathbf{H}\left(\mathbf{S} ; \mathbf{S}^{0}\right): \overline{\boldsymbol{\sigma}}\right\rangle_{\mathcal{C}}+\eta\left\langle\mathbf{G}\left(\mathbf{S}^{0}\right): \overline{\boldsymbol{\sigma}}\right\rangle_{\mathcal{O}},
$$

where the second-term on the r.h.s. represents the strain change due to the presence of grains, of volume fraction $0 \leq f \leq 1$, and the third-term on the r.h.s. the strain change due to the cracks. The contributions of individual grains (tensor $\mathbf{H}$ ) are averaged over all crystallographic orientations $\mathcal{C}$. Likewise, the contributions of the cracks (tensor $\mathbf{G}$ ) are averaged over all cracks orientations $\mathcal{O}$. The transversely-isotropic tensors $\mathbf{H}$ and $\mathbf{G}$ are obtained in terms of Eshelby's or Hill's tensor [25] for their respective problem. Tensor $\mathbf{H}$ reads (see e.g. [26]):

$$
\mathbf{H}\left(\mathbf{S} ; \mathbf{S}^{0}\right)=\left[\left(\mathbf{S}-\mathbf{S}^{0}\right)^{-1}+\mathbf{Q}\left(\mathbf{S}^{0}\right)\right]^{-1}
$$

in which $\mathbf{Q}$ is the isotropic stiffness tensor defined by [27, Chap. 2]:

$$
Q_{11}=Q_{22}=Q_{33}=\frac{16}{15} \frac{G^{0}}{1-\nu^{0}}, \quad Q_{12}=Q_{23}=Q_{13}=\frac{2 G^{0}}{15} \frac{1+5 \nu^{0}}{1-\nu^{0}}
$$


where we refer to the Voigt notation (7). Other components are obtained from the above, e.g. $Q_{44}=$ $Q_{55}=Q_{66}=\left(Q_{11}-Q_{12}\right) / 2$. The variables $G^{0}$ refer to the shear modulus associated to $\mathbf{S}^{0}$ and $\nu^{0}$ to its Poisson ratio, i.e. $G^{0}=1 / S_{44}^{0}$ and $\nu^{0}=S_{44}^{0} /\left(2 S_{11}^{0}\right)$ - 1. An equivalent form is given in [28] for the reciprocal tensor $\mathbf{P}=\mathbf{S}^{0}\left(\mathbf{I}-\mathbf{Q S}^{0}\right)$ whereas a more general expression for $\mathbf{Q}$ is given in [29] for an ellipsoidal inclusion with revolution symmetry.

Tensor G entering (13) is given in closed-form expression as [27, Chap. 5]:

$$
G_{33}=\frac{8\left(1-\nu^{0}\right)}{3 G^{0}}, \quad G_{44}=G_{55}=\frac{16\left(1-\nu^{0}\right)}{3 G^{0}\left(2-\nu^{0}\right)}, \quad G_{11}=G_{22}=G_{12}=G_{13}=G_{66}=0,
$$

in the Voigt notation (3) where $\mathrm{e}_{3}$ is normal to the crack.

Assume now that the embedding medium with compliance $\mathbf{S}^{0}$ has the same elastic response as the heterogeneous medium, so that $\mathbf{S}^{0}=\widetilde{\mathbf{S}}$. Accordingly, when the material is subjected to the macroscopic stress $\overline{\boldsymbol{\sigma}}$, the deformation induced by the cracks compensates that of the grains:

$$
\langle\mathbf{H}(\mathbf{S} ; \widetilde{\mathbf{S}})\rangle_{\mathcal{C}}+\eta\langle\mathbf{G}(\widetilde{\mathbf{S}})\rangle_{\mathcal{O}}=\mathbf{0},
$$

where we took $f=1$, so that the grains cover the entire domain and the cracks have no volume. The assumption $\mathbf{S}^{0}=\widetilde{\mathbf{S}}$ can be thought of as the construction of a hierarchical material with infinitely many scales, in the following way. Consider a material made of a homogeneous matrix containing widely-separated grains and cracks. Replace the embedding matrix by a set of grains and cracks at a much smaller length-scale, and repeat this operation until the matrix is entirely covered by grains and cracks. Now, in a polycrystal where cracks and grains have the same typical size, one may assume that each crack or grain is surrounded by many different, randomly-oriented cracks and grains. The approximation $\mathbf{S}^{0} \approx \widetilde{\mathbf{S}}$ amounts to replace the surrounding medium around each inhomogeneity by a homogeneous effective material.

Rewrite now Equation (17) using (14), (15) and (16). We refer to [29] for amenable formula for the product and inverse of transversely-isotropic tensors. The means $\langle\mathbf{A}\rangle_{\mathcal{C}, \mathcal{O}}$ of a transversely-isotropic stiffness tensor $\mathbf{A}$ with axis of symmetry parallel to $\mathrm{e}_{3}$ is the isotropic tensor with bulk and shear moduli [28]:

$$
K_{A}=\frac{2\left(A_{11}+A_{12}+2 A_{13}\right)+A_{33}}{9}, \quad G_{A}=\frac{3\left(2 A_{44}+A_{66}\right)+2\left(A_{11}-A_{13}\right)+A_{33}-A_{12}}{15},
$$

respectively, where the $A_{i j}$ refer to the Voigt notation (3). Denoting $K_{T}$ and $G_{T}$ the bulk and shear moduli associated to the compliance tensor of the l.h.s. of (17):

$$
\mathbf{T}=\langle\mathbf{H}(\mathbf{S} ; \widetilde{\mathbf{S}})\rangle_{\mathcal{C}}+\eta\langle\mathbf{G}(\widetilde{\mathbf{S}})\rangle_{\mathcal{O}}
$$

one obtains the expressions below for the inverse moduli:

$$
\begin{aligned}
K_{T}^{-1}= & \frac{2 u_{2}+u_{1}-4 u_{3}}{u_{1} u_{2}-2 u_{3}^{2}}+\frac{8 \eta(1-\widetilde{\nu})}{3 \widetilde{G}}, \quad \widetilde{\nu}=\frac{3 \widetilde{K}-2 \widetilde{G}}{2(3 \widetilde{K}+\widetilde{G})} \\
G_{T}^{-1} & =\frac{2 u_{2}+4\left(u_{1}+2 u_{3}\right)}{15\left(u_{1} u_{2}-2 u_{3}^{2}\right)}+\frac{6}{15 u_{4}}+\frac{6}{15 u_{5}}+\frac{32 \eta\left(5-6 \widetilde{\nu}+\widetilde{\nu}^{2}\right)}{45 \widetilde{G}(2-\widetilde{\nu})}
\end{aligned}
$$

where $\widetilde{\nu}$ is the effective Poisson ratio and the $u_{i}$ are a set of five independent components of the tensor 
$\mathbf{U}=\mathbf{H}^{-1}$ as follows:

$$
\begin{aligned}
u_{1}=U_{1111}+U_{1122}= & {\left[18 \widetilde{K} \widetilde{G}-2(\widetilde{G}+3 \widetilde{K}) E_{z}\right] \frac{E_{p} E_{z}}{\Delta_{u}}+\frac{2 \widetilde{G}(9+5 \widetilde{\nu})}{15(1-\widetilde{\nu})}, } \\
u_{2}=U_{3333}= & {\left[18 \widetilde{K} \widetilde{G}\left(1-\nu_{p}\right)-(4 \widetilde{G}+3 \widetilde{K}) E_{p}\right] \frac{E_{z}^{2}}{\Delta_{u}}+\frac{16 \widetilde{G}}{15(1-\widetilde{\nu})}, } \\
u_{3}=U_{1133}= & {\left[18 \widetilde{K} \widetilde{G} \nu_{z p}+(2 \widetilde{G}-3 \widetilde{K}) E_{z}\right] \frac{E_{p} E_{z}}{\Delta_{u}}+\frac{2 \widetilde{G}(1+5 \widetilde{\nu})}{15(1-\widetilde{\nu})}, } \\
u_{4}=U_{1212}= & \frac{\widetilde{G} E_{p}}{2 \widetilde{G}\left(1+\nu_{p}\right)-E_{p}}+\frac{\widetilde{G}(7-5 \widetilde{\nu})}{15(1-\widetilde{\nu})}, \\
u_{5}=U_{1313}= & \frac{\widetilde{G} G_{z p}}{\widetilde{G}-G_{z p}}+\frac{\widetilde{G}(7-5 \widetilde{\nu})}{15(1-\widetilde{\nu})}, \\
\Delta_{u}= & {\left[2\left(\nu_{p}-1\right) E_{z}+\left(8 \nu_{z p} \widetilde{\nu}-1\right) E_{p}\right](3 \widetilde{K}+\widetilde{G}) E_{z} } \\
& +18\left(1-\nu_{p}\right) \widetilde{K} \widetilde{G} E_{z}+3 E_{p}\left[E_{z}^{2}-\left(12 \widetilde{K} \nu_{z p}^{2}+E_{z}\right) \widetilde{G}\right],
\end{aligned}
$$

again, expressed in a basis where $e_{3}$ is the axis of transverse symmetry. The bulk and shear moduli $K_{T}$ and $G_{T}$ are, accordingly, rational functions of the bulk and shear moduli $\widetilde{K}$ and $\widetilde{G}$ and of all parameters of the problem. Condition (17) therefore can be rewritten as two polynomial equations in $\widetilde{K}$ and $\widetilde{G}$. The first one is of order 4 in $\widetilde{K}$ and 5 in $\widetilde{G}$, whereas the second one is of order 9 in $\widetilde{K}$ and 5 in $\widetilde{G}$. For an isotropic tensor $\mathbf{S}$ with bulk modulus $K$ and shear modulus $G$, they reduce to a system of cubic equations:

$$
\begin{aligned}
\widetilde{G} \widetilde{K}[(9+16 \eta) \widetilde{K}-9 K] & =3 \widetilde{G}^{2}(K-\widetilde{K})-12 \eta \widetilde{K}^{2} K \\
27(75-32 \eta) \widetilde{K}^{2} G=3 \widetilde{G} \widetilde{K}[27(25+16 \eta) \widetilde{K}+(704 \eta-675) G]+(450+512 \eta) \widetilde{G}^{3} & +3 \widetilde{G}^{2}[9(75+64 \eta) \widetilde{K}+2(128 \eta-75) G]
\end{aligned}
$$

Numerical solutions of the above are determined using the software "Mathematica" [30]. We obtained a unique set of real solutions in the domain $\widetilde{K}>0, \widetilde{G}>0$, for all elastic moduli $\widetilde{K}$ and $\widetilde{G}$ and crackdensity parameter $\eta$ considered in the present work. For arbitrary anisotropy, numerical solutions are readily obtained using the following fixed-point algorithm:

$$
\begin{aligned}
& \text { Initialization: } \text { set } \widetilde{K}^{-1}=9 \Delta /\left[2 E_{p}+\left(1-\nu_{p}+4 \nu_{p z}\right) E_{z}\right] \\
& \qquad \widetilde{G}^{-1}=6 \Delta /\left[E_{p}+2\left(1-\nu_{p}-2 \nu_{p z}\right) E_{z}\right] s, \quad \Delta=1-\nu_{p}-2 \nu_{p z} \nu_{z p} . \\
& \text { Iterations: } \quad \text { set } \widetilde{K}^{-1} \leftarrow \widetilde{K}^{-1}+K_{T}^{-1}(\widetilde{K}, \widetilde{G}), \quad \widetilde{G}^{-1} \leftarrow \widetilde{G}^{-1}+G_{T}^{-1}(\widetilde{K}, \widetilde{G}), \\
& \text { until the absolute values of } \widetilde{K} \text { and } \widetilde{G} \text { change by less than } 10^{-10} \text {. }
\end{aligned}
$$

In this algorithm, the effective moduli are initialized to the Voigt-Hill upper-bound given by $\left\langle\mathbf{S}^{-1}\right\rangle_{\mathcal{C}}$ (step 23a) whereas iterations (23b) enforce infinite bulk and shear moduli $K_{T}=G_{T}=\infty$, i.e. the compliance tensor $\mathbf{T}$ vanishes. We emphasize that the value of $\widetilde{K}$ used to evaluate successively the functions $K_{T}$ and $G_{T}$ in step (23b) changes, as $\widetilde{K}$ is updated before $\widetilde{G}$. No convergence is observed, except at small cracks-density values, when the same value of $\widetilde{K}$ is used to evaluate $K_{T}$ and $G_{T}$. With the above algorithm, nevertheless, we obtained fast convergence for arbitrary crack-density $\eta$ and all sets of crystal anisotropy considered in the present study.

To estimate the effective thermal expansion tensor, we rely on the exact relationship [31]:

$$
\widetilde{\boldsymbol{\alpha}}:\langle\boldsymbol{\sigma}(\boldsymbol{x})\rangle=\langle\boldsymbol{\alpha}(\boldsymbol{x}): \boldsymbol{\sigma}(\boldsymbol{x})\rangle,
$$

where $\boldsymbol{\sigma}(\boldsymbol{x})$ is the local stress field resulting from the macroscopic stress loading $\langle\boldsymbol{\sigma}(\boldsymbol{x})\rangle=\overline{\boldsymbol{\sigma}}$, with no 
thermal loading applied $(\Delta T=0)$. Since the stress is distributed over all grains, the l.h.s. and r.h.s. may be rewritten as a mean over all crystallographic orientations $i$ :

$$
\widetilde{\boldsymbol{\alpha}}:\left\langle\boldsymbol{\sigma}^{i}\right\rangle_{i}=\left\langle\boldsymbol{\alpha}^{i}: \boldsymbol{\sigma}^{i}\right\rangle_{i}
$$

where $\boldsymbol{\sigma}^{i}$ is the constant stress field occuring in an isolated spherical inclusion of compliance tensor $\mathbf{S}^{i}$ and thermal expansion tensor $\boldsymbol{\alpha}^{i}$, oriented along direction $i$, and embedded in a medium with compliance tensor $\mathbf{S}^{0}$. Now, the macroscopic strain $\bar{\varepsilon}$ in a large domain containing one single inclusion can be decomposed as:

$$
\bar{\varepsilon}=\mathbf{S}^{0}:\left(\overline{\boldsymbol{\sigma}}-f_{i} \boldsymbol{\sigma}^{i}\right)+f_{i} \mathbf{S}^{i}: \boldsymbol{\sigma}^{i},
$$

where $f_{i}$ is the inclusion volume fraction. Comparing the above to (13), one obtains $\boldsymbol{\sigma}^{i}=\boldsymbol{\Gamma}: \overline{\boldsymbol{\sigma}}$ where the tensor $\boldsymbol{\Gamma}=\left[\mathbf{I}+\mathbf{Q}:\left(\mathbf{S}-\mathbf{S}^{0}\right)\right]^{-1}$ allows one to express the uniform field inside the inhomogeneity in terms of the prescribed field (see e.g. [29]). Equation (25) now reduces to:

$$
\widetilde{\boldsymbol{\alpha}}:\langle\boldsymbol{\Gamma}\rangle_{\mathcal{C}}: \overline{\boldsymbol{\sigma}}=\langle\boldsymbol{\alpha}: \boldsymbol{\Gamma}\rangle_{\mathcal{C}}: \overline{\boldsymbol{\sigma}}
$$

and, taking $\bar{\sigma}_{i j}=\delta_{i j}$ :

$$
\widetilde{\alpha}_{V}=\frac{6 \alpha_{11}\left(\Gamma_{1111}+\Gamma_{1122}+\Gamma_{1133}\right)+3 \alpha_{33}\left(\Gamma_{3333}+2 \Gamma_{3311}\right)}{\Gamma_{3333}+2\left(\Gamma_{1111}+\Gamma_{1122}+\Gamma_{1133}+\Gamma_{3311}\right)},
$$

where the components of $\boldsymbol{\Gamma}$ are given in a basis with axis of symmetry $\mathrm{e}_{3}$. Making use of (15) the effective thermal expansion coefficient reads:

$$
\begin{aligned}
\frac{\widetilde{\alpha}_{V}}{3} & =\frac{\alpha_{11} \gamma_{z}+\alpha_{33} \gamma_{p}}{\gamma_{z}+\gamma_{p}}, \quad \Delta_{\gamma}=\frac{\widetilde{\nu}}{2 \widetilde{G}(1+\widetilde{\nu})}-\frac{\nu_{z p}}{E_{z}}, \\
\gamma_{z} & =\left(2 u_{3}-u_{1}\right) \Delta_{\gamma}+\left(u_{2}-u_{3}\right)\left[\frac{1}{E_{z}}-\frac{1}{2 \widetilde{G}(1+\widetilde{\nu})}\right], \\
\gamma_{p} & =\left(u_{3}-u_{2}\right) \Delta_{\gamma}+\left(u_{1}-2 u_{3}\right)\left[\frac{1-\nu_{p}}{2 E_{p}}-\frac{1-\widetilde{\nu}}{4 \widetilde{G}(1+\widetilde{\nu})}\right],
\end{aligned}
$$

where the $u_{i}$ are given in Equation (21), and the effective bulk and shear moduli $\widetilde{K}$ and $\widetilde{G}$ are the solutions of (17).

In what follows, the self-consistent estimates (17) and (28) are denoted $\left(\mathrm{SC}^{\prime}\right)$. When the polycrystal contains no crack $(\eta=0)$, the system (17) reduces to two polynomial equations, one of degree 2 in $\widetilde{K}$ and the other of degree 6 in $\widetilde{G}$ (not shown here). Using the software Mathematica [30], the authors verified that these two polynomial equations and the self-consistent estimate of Berryman [32] have the same unique roots in the domain $\widetilde{K}>0$ and $\widetilde{G}>0$. Accordingly, in the absence of cracks, our approach is equivalent to that of Berryman's for elasticity. Berryman's estimates are based on HashinShtrikman bounds for the polycrystal (not considered in the present approach) and are determined by the equations:

$$
\begin{aligned}
3 G_{V} & =C_{33}+C_{11}-2 C_{13}-C_{66}, \quad 3 K_{V} G_{R}=C_{33}\left(C_{11}-C_{66}\right)-C_{13}^{2}, \\
9 K_{V} & =C_{33}+2 C_{11}+2 C_{12}+4 C_{13}, \quad \xi=\frac{\widetilde{G}}{6} \frac{9 \widetilde{K}+8 \widetilde{G}}{\widetilde{K}+2 \widetilde{G}}, \\
\frac{5}{\widetilde{G}+\xi} & =\frac{K_{V}+\frac{4}{3} G}{\left(\widetilde{K}+\frac{4}{3} \widetilde{G}\right)\left(G_{V}+\xi\right)}+\frac{2}{C_{44}+\xi}+\frac{2}{C_{66}+\xi}, \quad \widetilde{K}=K_{V} \frac{G_{R}+\xi}{G_{V}+\xi},
\end{aligned}
$$

where the $C_{i j}$ refer to the Voigt notation (7) for the stiffness tensor of the hexagonal crystal. 
Consider now the exact cross-property relation, due to Hashin [33], which relates the bulk and volumetric thermal expansion coefficient of isotropic polycrystalline aggregates containing hexagonal crystals such that $\mathbf{S}$ and $\boldsymbol{\alpha}$ share a common axis of symmetry:

$$
\widetilde{\alpha}_{V}=\widetilde{\alpha}_{11}+\widetilde{\alpha}_{22}+\widetilde{\alpha}_{33}=\alpha_{V}+\left(\alpha_{33}-\alpha_{11}\right) \frac{1 / \widetilde{K}-S_{i i k k}}{S_{i i 33}-S_{i i 11}}
$$

where $e_{3}$ is the axis of symmetry of the crystal and repeated indices are to be summed up. Hashin's result applies to the present problem when $\eta=0$, and as expected estimates (17) and (28) satisfy (30) in that case. When $\eta \neq 0$ instead, (30) is not verified.

\section{Percolating behavior}

In porous and rigidly-reinforced materials, self-consistent estimates typically predict a percolation threshold. The results of these methods should be taken with care, especially with respect to percolating behavior, where the theory's predictions are sometimes unrealistic compared to real materials [34]. In linear elasticity, for instance, different percolation thresholds may be obtained for the bulk and shear moduli - whereas in real materials, the mechanical and geometrical percolation thresholds are expected to coincide (see [35, Chap. 10] for a discussion). This is because self-consistent estimates are realizable by very peculiar, hierarchical microstructures made of infinitely-many scales [36] and with no finite correlation length [37]. That being said, it is nevertheless a merit of the self-consistent theory that, in contrast with most other approximations and bounds, it does predict a percolation threshold (see e.g. [38] for a discussion).

For cracked media, the percolation thresholds is often expressed in terms of a critical crack-density threshold $\eta_{c}$, as, for instance, in the context of conductivity [39], permeability [40] or elastic wave propagation [41]. In the present problem of quasi-static elasticity, the percolation threshold is associated to the loss of connectivity of the matrix [42], at which point the effective elastic moduli vanish. For a Boolean set of uniformly-oriented disks, the geometrical percolation threshold of the complementary set of the disks has been estimated numerically in [43]. The authors give the threshold $(4 / 3) \pi \eta_{c} \approx 22.9$ i.e. $\eta_{c} \approx 5.5$ for the critical crack-density.

The zeros of the Euler-Poincaré characteristic (or connectivity number) has also been used to estimate the percolation thresholds of binary media [44]. Its expession reads, for a Boolean model of volume fraction $1-q$, intensity $\theta$ and primary grain $A$ :

$$
q \theta\left(1-\frac{\theta M_{A} S_{A}}{4 \pi}+\frac{\pi \theta^{2} S_{A}^{3}}{384}\right)
$$

where $S_{A}$ is the surface area of $A$ and $M_{A}$ the integral of the mean curvature along the boundary of $A$. For a disk of radius $a, S_{A}=2 \pi a^{2}, M_{A}=\pi^{2} a$ so that, setting the above expression to zero provides us with the approximation $\eta_{c}=\theta a^{3}=\left(4 / \pi^{2}\right) \times(3 \pm \sqrt{6})$. The first value $\eta_{c} \approx 0.2231$ is close to the numerical estimate $\eta_{c} \approx 0.2230$ [45] for the percolation threshold of the disks. However the prediction using the connectivity number for the second threshold, $\eta_{c} \approx 2.21$, related to the loss of connectivity of the matrix, is significantly smaller than the estimate given in [43]. For linear elasticity, the selfconsistent scheme of Budiansky and O'Conell [5] predicts the percolation threshold $\eta_{c}=9 / 16 \approx 0.56$ for "dry cracks" embedded in an isotropic homogeneous body, whereas a different percolation threshold $\eta_{c}=45 / 32 \approx 1.41$ is obtained for "wet" (fluid-saturated) cracks.

For the self-consistent estimates proposed in this work, the percolating behavior is obtained by expanding $K_{T}^{-1}$ and $G_{T}^{-1}$ (Equation 20) as a Taylor series of order 2 near the point $\eta \rightarrow \eta_{c}$ where $\eta_{c}$ is unknown. The equations $K_{T}^{-1}=0$ and $G_{T}^{-1}=0$ provide a set of conditions on $\widetilde{K}, \widetilde{G}$ and $\eta_{c}$. We look for solutions of the form $\widetilde{K} \sim K_{1}\left(\eta_{c}-\eta\right), \widetilde{G} \sim G_{1}\left(\eta_{c}-\eta\right)$ where the prefactors $K_{1}$ and $G_{1}$ are independent of $\eta$. These asymptotic forms will be confirmed later on in this study, using numerical 
results. We obtain the following condition on $\eta_{c}$ :

$$
135\left(225+1424 \eta_{c}\right)=\left(64 \eta_{c}\right)^{2}\left(64 \eta_{c}-28\right),
$$

which provides the percolation threshold $\eta_{c} \approx 1.15752$. Furthermore, in the limit $\eta \rightarrow \eta_{c}$, the selfconsistent estimates predicts constant thermal expansion coefficient and Poisson ratio, independently of the crystal's elastic moduli:

$$
\widetilde{\alpha}_{V} \rightarrow \alpha_{V}=2 \alpha_{11}+\alpha_{33}, \quad \widetilde{\nu} \rightarrow \frac{8704 \eta_{c}^{2}-4608 \eta_{c}-6075}{512 \eta_{c}^{2}+1800 \eta_{c}} \approx 0.09144
$$

The limiting value for $\widetilde{\alpha}_{V}$ implies, in light of (24):

$$
\langle\boldsymbol{\sigma}(\boldsymbol{x}): \boldsymbol{\alpha}(\boldsymbol{x})\rangle=\langle\boldsymbol{\sigma}(\boldsymbol{x})\rangle:\langle\boldsymbol{\alpha}(\boldsymbol{x})\rangle,
$$

where $\boldsymbol{\sigma}(\boldsymbol{x})$ is the local stress field occurring in the polycrystalline material subjected to stress loading, with no thermal loading. The above equality can be interpreted as a lack of correlation between the crystal's orientations and the stress field, as the percolation threshold is approached.

The considerations leading to (32) do not hold when some of the eigenvalues of $\mathbf{C}$ vanish. We first investigate the case $G_{z p}=0$ with the moduli $E_{z}$ and $E_{p}$ strictly positive. Equation (20) yields the lower percolation threshold $\eta_{c}^{\prime}$ solution of:

$$
4\left(2048 \eta_{c}^{\prime 2}+2448 \eta_{c}^{\prime}+675\right)=\left(2176 \eta_{c}^{\prime}+1125\right) \sqrt{16 \eta_{c}^{\prime}+9}
$$

so that $\eta_{c}^{\prime} \approx 0.373126$. Second, when $E_{z}=0$, keeping $G_{z p}>0, E_{p}>0$, a third percolation threshold $\eta_{c}^{\prime \prime}$, in-between $\eta_{c}^{\prime}$ and $\eta_{c}$ is obtained:

$$
\eta_{c}^{\prime \prime}=\frac{32+13 \sqrt{46}}{160} \approx 0.751064
$$

In Figs. (1) and (2), we compare the predictions of estimates (22a) with three homogenization schemes developped in the litterature: the self-consistent scheme of Budiansky and O'Connell [5] (denoted SC), the Mori-Tanaka method of Benveniste [46] (denoted MT) and the Hashin-Shtrikman-type bounds of Ponte Castañeda and Willis [47] (denoted HS). The effective Young modulus $\widetilde{E}$ is represented in Figs. (1) as a function of $\eta$. Scheme (MT) amounts to using an asymptotic expansion truncated to first-order in $\eta \ll 1$ for the inverse $1 / \widetilde{E}$, and is equivalent to the "non-interacting approximation" presented in [26]. Notice that the scales used for coordinate $\eta$ (horizontal axis) are quite different for the two self-consistent estimates $\mathrm{SC}^{\prime}$ and $\mathrm{SC}$ (Figs. 1a and 1d).

Method (HS) provides rigorous upper-bounds for classes of materials containing cracks embedded in uncracked regions ("security spheres"). In this construction, the cracks do not interpenetrate and the cracks-density parameter is constrained in the range $\eta \leq 3 /(4 \pi) \approx 0.239$. The four methods are equivalent up to the first-order correction in $\eta$ in the limit $\eta \rightarrow 0$, and are qualitatively similar for small cracks-density parameter $\eta \leq 0.4$. The stiffest estimate is provided by scheme MT which percolates at $\eta=\infty$, whereas method $\mathrm{SC}$ is the most compliant. This behavior is a consequence of the self-consistent assumption employed in method SC, which reads [6]:

$$
\widetilde{\mathbf{S}}=\mathbf{S}+\eta\langle\mathbf{G}(\widetilde{\mathbf{S}})\rangle_{\mathcal{O}}
$$

This is in contrast with the present method, where both the matrix phase and the cracks are embedded in the equivalent medium.

Method (HS) predicts different percolation thresholds depending on $\nu$, however these thresholds lie outside of the domain of validity of the bounds. The present method provides a material-independent 


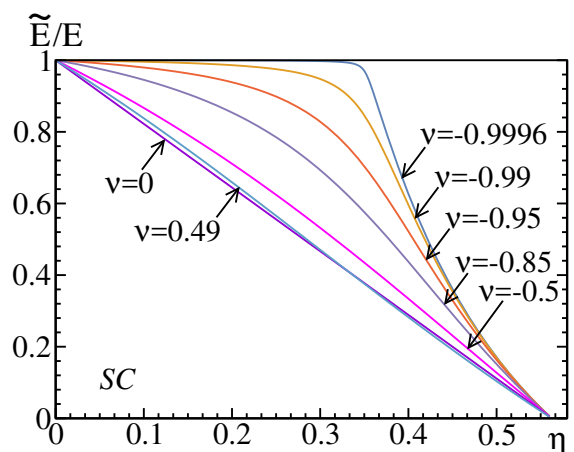

(a)

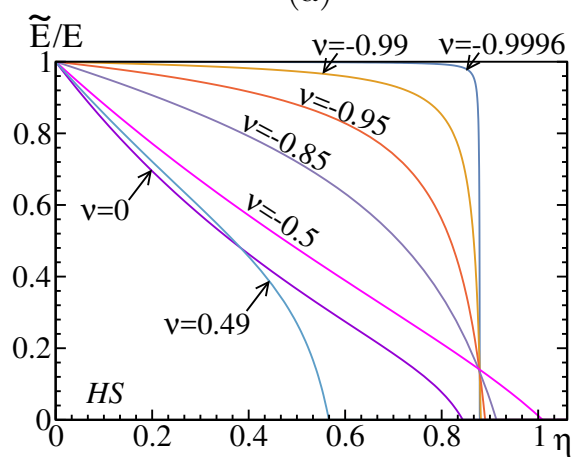

(c)

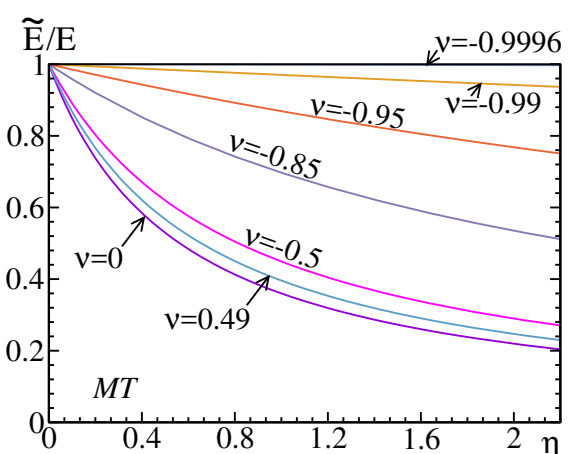

(b)

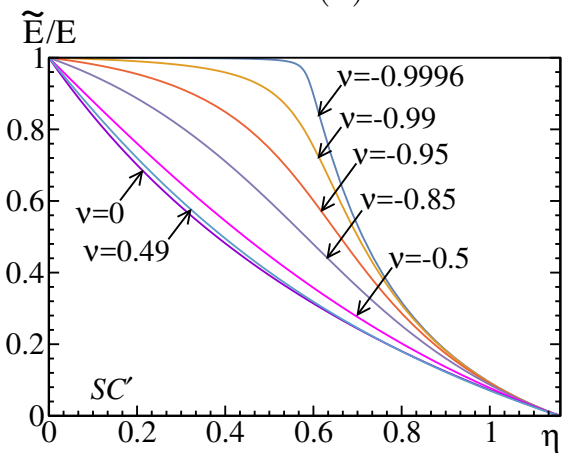

(d)

Figure 1. Normalized effective Young modulus $\widetilde{E} / \widetilde{E}$ vs. crack density $\eta$ for a homogeneous isotropic solid containing randomlyoriented cracks, for various Poisson ratios $\nu$ in the matrix: estimates SC (a) and MT (b), bounds HS (c), present self-consistent method (d).

percolation threshold at about $\eta \approx 1.16$, consistently with $(32)$. The effective Poisson ratio $\widetilde{\nu}$ is represented in Figs. (2) as a function of $\eta$ for various Poisson ratios $\nu$ in the matrix (the same values as in Fig. 1 are used). The limiting value for the effective Poisson ratio at percolation (Equation 33, method $\left.\mathrm{SC}^{\prime}\right)$ is confirmed in Fig. (2d)

\section{Comparison with Fourier-based numerical results}

\subsection{Isotropic body containing cracks}

In the following, we compare the predictions of $\mathrm{SC}^{\prime}$ with Fourier-based numerical computations. We first consider the simple Poisson-Voronoi tessellation for the polycrystalline aggregate, parametrized by the density of germs, equal to $n_{g} / V$, the number of grains per unit volume. Assuming the volume $\Omega$ is a cube of dimension $L$, we choose to parametrize the tessellation by the grains typical size $\ell=\left[3 /\left(4 \pi / n_{g}\right)\right]^{1 / 3} L$, defined so that the mean grain volume equals that of a sphere of radius $\ell$. We model the cracks by a Boolean model of equisized disks of radius $a$ with homogeneous Poisson point process. The intensity of the Poisson point process is $\eta a^{3}$. The Boolean model of disks is "superimposed" on the tessellation, so that the orientations of the grains and cracks, and their position compared to one another, are uncorrelated. In method $\mathrm{SC}^{\prime}$, no scale-separation hypothesis is made between the typical length scales of the cracks and that of the grains. Hereafter we choose $a=\ell$ so that the cracks and grains have similar size. A schematic view of the model is presented in Fig. (3). The Voronoï-Boolean model is not representative of real materials, but is useful nevertheless to draw comparisons between analytical estimates and exact numerical results.

Numerical Fourier-based computations are carried out on the random cracked-polycrystal model, using the backward-and-forward difference scheme [48, 49]. The domain $\Omega$ is discretized on grids of $L^{3}=128^{3}, 256^{3}$ and $512^{3}$ voxels, the cylindrical cracks have a diameter of $D=40$ or $D=80$ voxels 


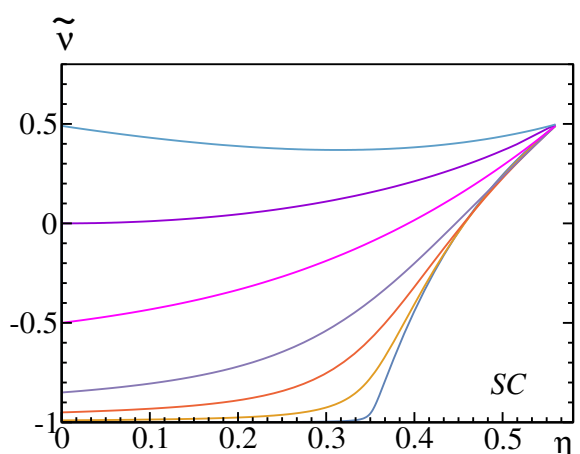

(a)

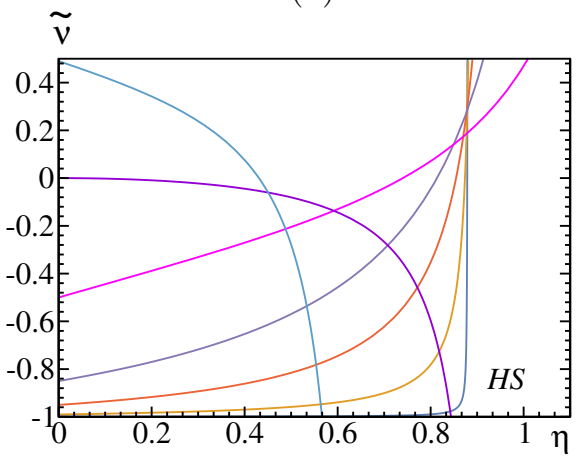

(c)

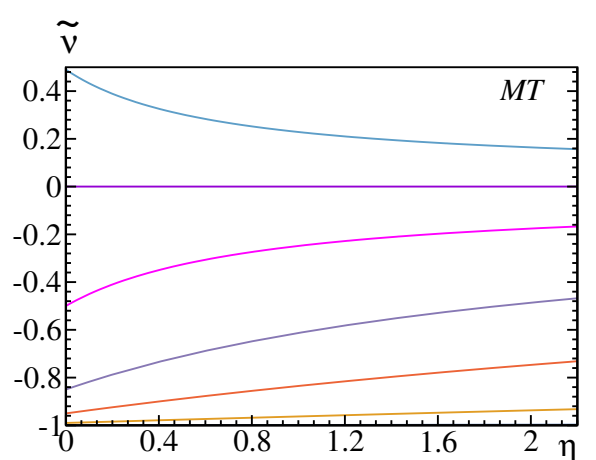

(b)

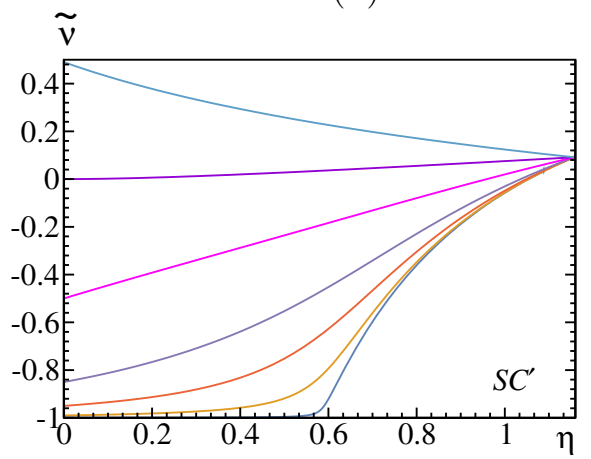

(d)

Figure 2. Effective Poisson ratio $\widetilde{\nu}$ vs. crack density $\eta$ for a homogeneous isotropic solid containing randomly-oriented cracks, for various Poisson ratios $\nu$ in the matrix: estimates SC (a) and MT (b), bounds HS (c), present self-consistent method (d).

and the thickness is set to $w=1.25,1.4,1.5,2,3,4$ and 5 voxels. The diameter of the cracks in each configuration is adjusted so that the crack-density parameter stay the same. As described in another study [50], the effective elastic moduli are overestimated when $w \leq 1.4$, whereas a linear behavior is observed with resprect to $w$ in the region $w \geq 1.5$. Results for all data sets are extrapolated in the limit $w \rightarrow 0$. The mean absolute difference between the apparent moduli computed for the various data sets, i.e. different values of $L$ and $D$, are used to estimate error bars. Data detailed in [50] show that the error on the bulk modulus is not more than $1 \%$ when $\eta \approx 0.38$. Nevertheless, the precision of the method deteriorates when the number of cracks increases, and hereafter we restrict ourselves to $\eta \leq 0.8$.

FFT predictions for the normalized bulk modulus $\widetilde{K} / K$ and normalized shear modulus $\widetilde{G} / G$ are shown in Fig. (4), and compared to the analytical estimates SC, $\mathrm{SC}^{\prime}, \mathrm{MT}$ and HS. As discussed previously, the analytical estimates differ significantly. Numerical results for the Boolean microstructure

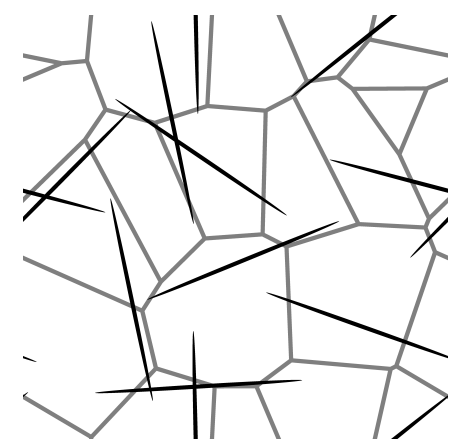

Figure 3. Random model of polycrystal containing a population of cracks: Poisson-Voronoï tessellation superimposed on a Boolean model of penny-shaped cracks (2D cut). 


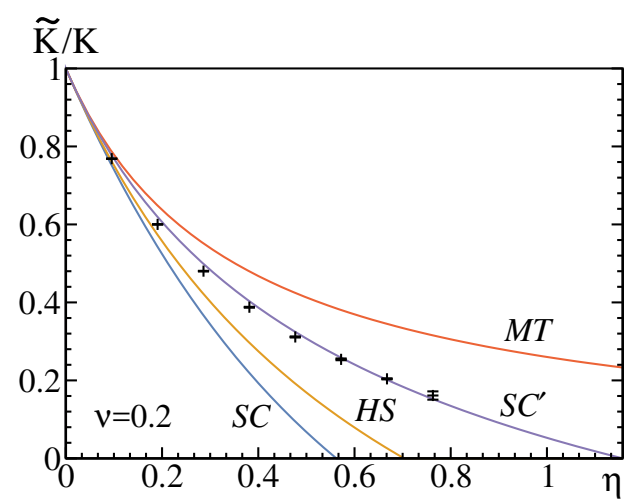

(a)

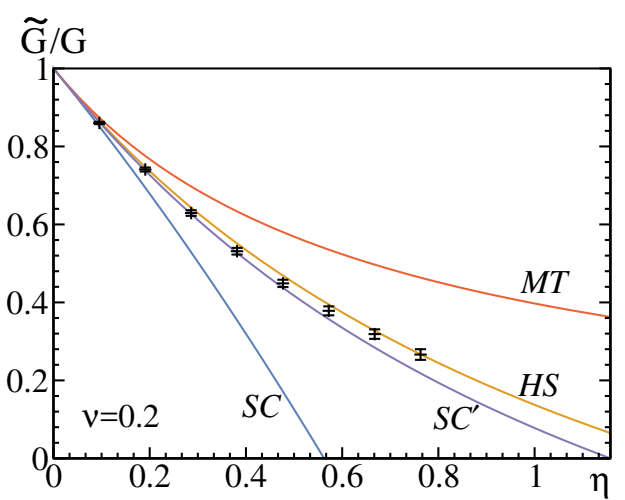

(b)

Figure 4. Normalized bulk modulus $\widetilde{K} / K($ a) and shear modulus $\widetilde{G} / G$ (b) vs. crack density $\eta$ for a homogeneous isotropic body (Poisson ratio 0.2) containing cracks: comparison between analytical methods SC, SC', HS, MT and FFT numerical results.

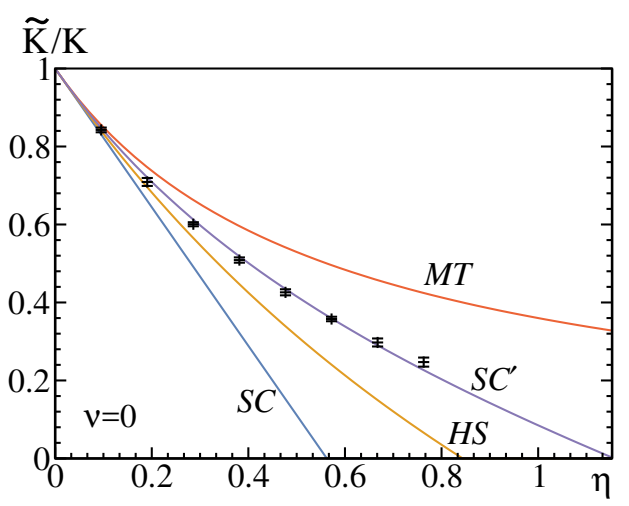

Figure 5. Normalized bulk modulus $\widetilde{K} / K$ vs. crack density $\eta$ for a homogeneous isotropic body (Poisson ratio 0 ) containing cracks: comparison between analytical methods SC, SC', HS, MT and FFT numerical results.

considered here are very close to the predictions of methods $\mathrm{SC}^{\prime}$, up to about $\eta \approx 0.6$. At higher crack density $(\eta \geq 0.6)$, the estimates provided by method $\mathrm{SC}^{\prime}$ are more compliant than numerical results. This behavior is not surprising since the percolation threshold for the loss of connectivity of the matrix, equal to 5.46 according to Yi and Esmail [43], is much higher than $\eta_{c} \approx 1.16$. Similar observations hold when the Poisson ratio is $\nu=0$ in the matrix (Fig. 5).

\subsection{Cracked polycrystalline aggregates}

Method $\mathrm{SC}^{\prime}$ is now applied to a polycrystal containing cracks. To illustrate the predictions of the method, we fix $E_{z}=1, G_{z p}=5 / 12, \nu_{z p}=\nu_{p}=0.2$ and let $E_{p}$ vary between 0 to its maximum value 10 (see Equation 6). Isotropic crystals are recovered when $E_{p}=E_{z}=1$ (black solid line). The effective thermoelastic properties are represented as a function of the cracks density $\eta$ in Fig. (6). The limiting value of $\widetilde{\nu} \approx 0.091$ (Equation 33) is recovered when $\eta \rightarrow \eta_{c}$, for all values of $E_{p}$, as well as $\widetilde{\alpha}_{V}=\alpha_{V}$. In Fig. (6e) we have choosen $\alpha_{11}=1$ and $\alpha_{33}=0$, however the behavior $\widetilde{\alpha}_{V}$ for any value of $\alpha_{11}$ and $\alpha_{33}$ can be obtained from this data.

The onset of an "advanced" percolation threshold when $G_{z p} \rightarrow 0$ (Equation 35) is confirmed in Fig. (8a), where the effective bulk modulus $\widetilde{K}$ is represented as a function of $\eta$ for decreasing values of $G_{z p}$ monitored by the parameter $\gamma=G_{z p} /(5 / 12)=0.50,0.1,0.01,10^{-3}$ and 0 . At $G_{z p}=0, \mathrm{SC}^{\prime}$ predicts vanishing bulk and shear moduli when $\eta \geq \eta_{c}^{\prime} \approx 0.37$. Likewise, in the limit $E_{z} \rightarrow 0$ a percolation threshold $\eta_{c}^{\prime \prime} \approx 0.75$ (Equation 36) is obtained (Fig. 7).

Additionally, in the case $G_{z p} \rightarrow 0$, we have carried out numerical FFT computations (see symbols 


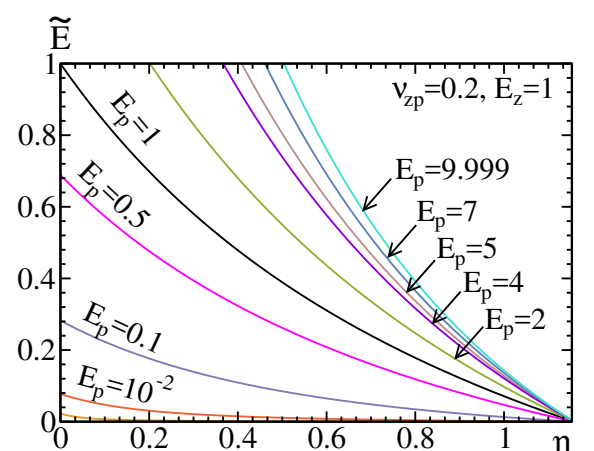

(a)

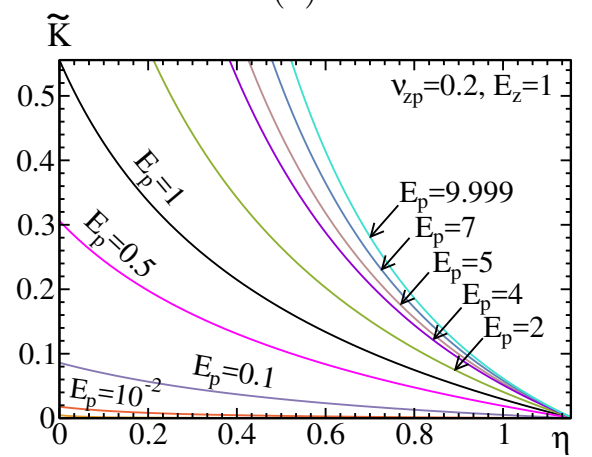

(c)

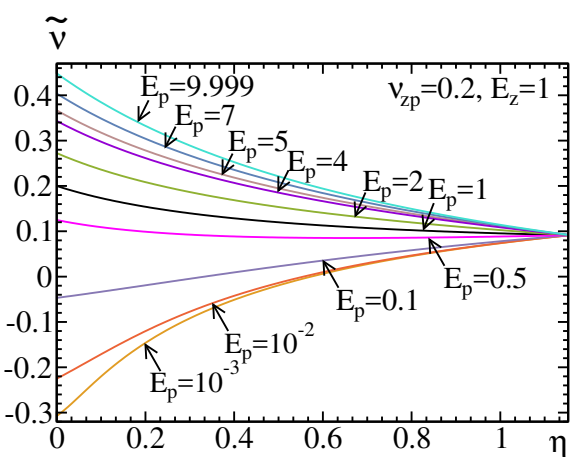

(b)

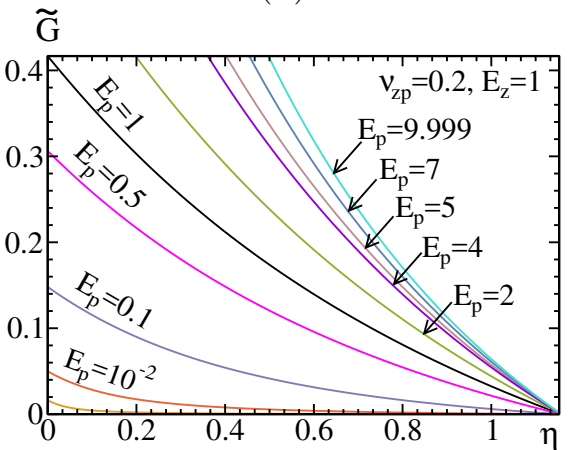

(d)

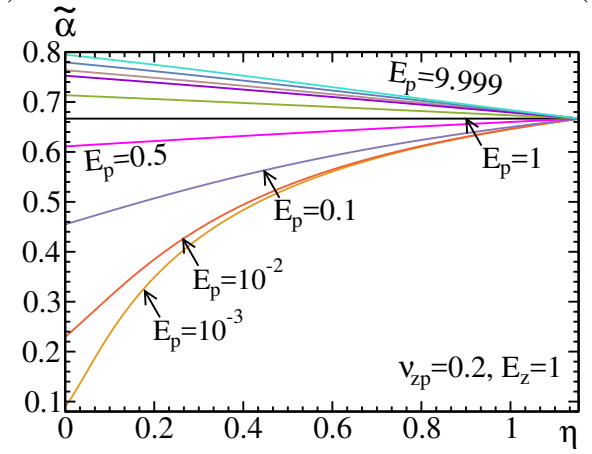

(e)

Figure 6. Self-consistent estimate $\mathrm{SC}^{\prime}$ for the effective response of a cracked polycrystal vs. crack density $\eta$, for varying in-plane Young modulus $E_{p} \leq 10$ with $\nu_{z p}=0.2, E_{z}=1, \alpha_{11}=1$ and $\alpha_{33}=0$. (a) Young modulus $\widetilde{E}$, (b) Poisson ratio $\widetilde{\nu}$, (c) bulk modulus $\widetilde{K}$, (d) shear modulus $\widetilde{G}$, (e) thermal expansion coefficient $\widetilde{\alpha}=\widetilde{\alpha}_{V} / 3$. 
in Fig. 8a). The lie very close to the predictions of $\mathrm{SC}^{\prime}$ when the anisotropy is moderate $\left(G_{z p}>0.1\right)$ or when $\eta$ is smaller than $\eta_{c}^{\prime}$. This is not the case when the crack density is high $\left(\eta>\eta_{c}^{\prime}\right)$ and the out-of-plane shear modulus is very small $\left(G_{z p} \leq 0.01\right)$. On the one hand, FFT results indicate a finite limit for the effective elastic moduli of the cracked polycrystals, even when $G_{z p}=0$. This is confirmed in Fig. (8b) which represents the effective bulk modulus as a function of $G_{z p}$, in semi-log plot, when $\eta=0.3815$ is fixed. In this plot, the dotted line is the FFT prediction when $G_{z p}$ is strictly zero. On the other hand, although no advanced percolation threshold is observed according to FFT results, a strong decrease of the effective moduli with respect to $\eta$ is observed up to about 0.4 , followed by a slower decrease in the range $\eta>0.4$.

These results suggest the existence of a mechanism responsible for the strong weakening of the moduli at moderate crack density. The local stress fields are useful to interpret this phenomenon. The stress component $\sigma_{33}$ occuring in a crystal is represented in Fig. (10), with cracks shown in white. The crystal has been choosen so that its axis of symmetry, $\mathrm{e}_{3}$, lies almost exactly in the same plane as the $2 \mathrm{D}$ cut where the fields are represented. Accordingly, $\sigma_{33}$ corresponds to the traction component in the out-of-plane direction, and is oriented about 45 degrees from the horizontal in Fig. (10). Maps (a) to (e) show the fields at decreasing values of $G_{z p}$. When the shear modulus $G_{z p}$ approaches zero, the material is weakened by large regions where the stress field $\sigma_{33}$ is close to zero (Fig. (10e, regions in blue). These regions organize through large bands parallel to direction es. Indeed, $G_{z p}=0$ implies in the crystal $\sigma_{31}=\sigma_{32} \equiv 0$. Accordingly, using stress equilibrium, $\partial_{3} \sigma_{33} \equiv 0$. Along cracks embedded in the crystal, however, $\boldsymbol{\sigma} \cdot \boldsymbol{n}=0$ implies $\sigma_{33} n_{3}=0$. These two results explain the development of weakening bands parallel to direction $\mathrm{e}_{3}$, enclosing cracks. The weakening zones however do not cross the grain boundary (not shown). When the crystals are not covered by such bands, an increase of $\eta$ has a strong weakening effect as each crack will weaken large regions in the surrounding crystals. When most of the material is already covered by weakening bands, however, an increase of $\eta$ has a much smaller effect. Weakening bands have been previously observed in $2 \mathrm{D}$ periodic structures with strongly-anisotropic response [51]. The authors of this study show that the typical length of the bands increase with the material anisotropy. In the polycrystalline materials considered here, the weakening bands do not cross grain boundaries.

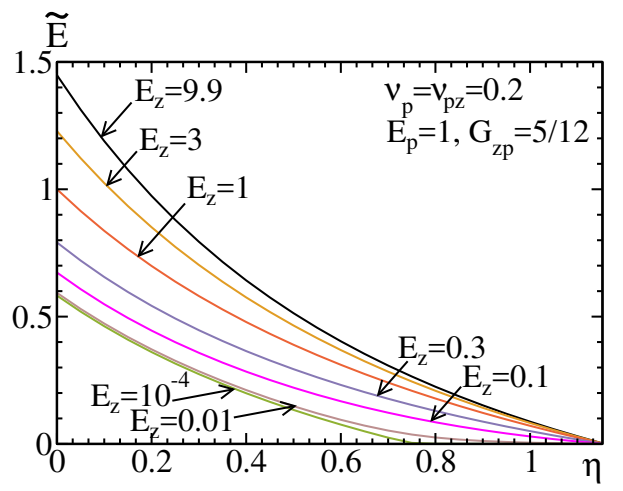

Figure 7. Effective Young modulus $\widetilde{E}$ as a function of the crack-density parameter $\eta$, for decreasing values of $E_{z}$.

The effect of scale separation is illustrated in Fig. (9), which shows the effective bulk modulus of cracked polycrystals as a function of the crack density $\eta$, for various values of the anisotropy ratio $\gamma=G_{z p} / G_{0}$. The value $\gamma=1$ corresponds to an isotropic tensor $\mathbf{C}$ with Young moulus 1 and Poisson ratio 0.2. Two configurations are considered. In the first one, modeled using $\mathrm{SC}^{\prime}$, the cracks and grains have similar size, as previously considered (solid lines). In the second one, the size of the cracks is much larger than that of the grains (dotted lines). This latter case is modeled using method $\mathrm{SC}^{\prime}$ two times. The matrix surrounding the crack is first replaced by an effective medium by applying $\mathrm{SC}^{\prime}$ to an uncracked polycrystal (in this case the method is equivalent to Berryman's). Second, the cracks are accounted for using method $\mathrm{SC}^{\prime}$ specialized to a homogeneous body containing cracks (Eq. 22a). The two estimates coincide only when the crystals are isotropic $(\gamma=1$, black line). In all other cases, the elastic response is stiffer when the cracks are larger than the grains. If scale separation is 


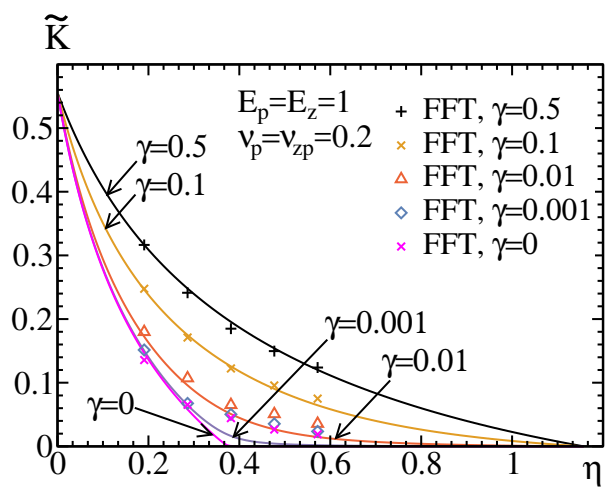

(a)

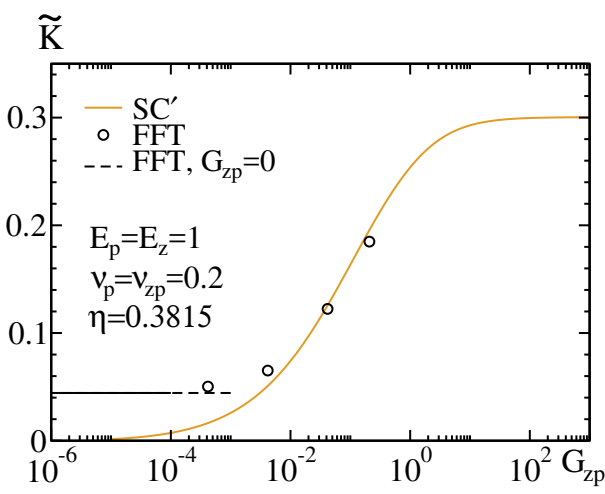

(b)

Figure 8. (a) Bulk modulus $\widetilde{K}$ as a function of $\eta$ for decreasing values of the out-of-plane shear modulus $G_{z p}$, monitored by the anisotropy ratio $\gamma=G_{z p} / G_{0}$ with $G_{0}=5 / 12$. (b) Bulk modulus $\widetilde{K}$ as a function of $G_{z p}$ at $\eta=0.3815$ in lin-log plot. In the two plots, FFT predictions are compared to method $\mathrm{SC}^{\prime}$.

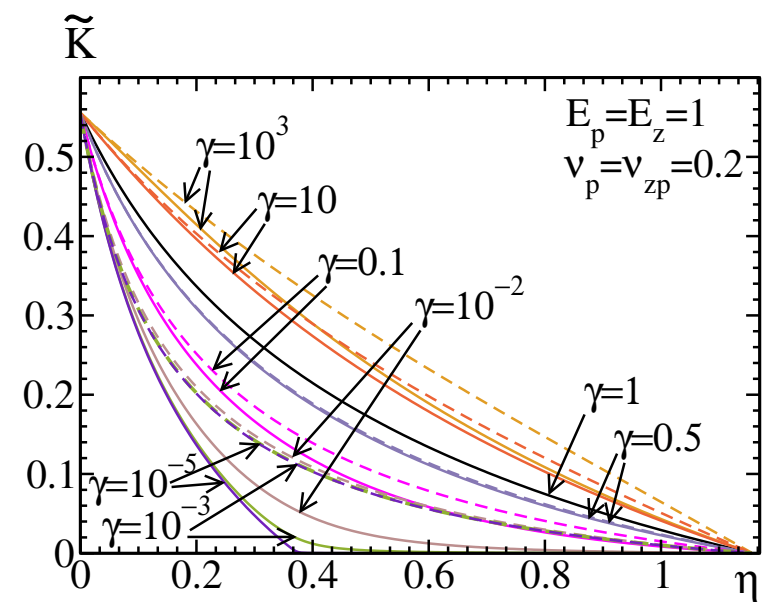

Figure 9. Effect of scale separation on the elastic response of a cracked polycrystal as a function of $\eta$ : effective bulk modulus $\widetilde{K}$ at increasing values of the out-of-plane shear modulus $G_{z p}$, monitored by the anisotropy ratio $\gamma=G_{z p} / G_{0}\left(G_{0}=5 / 12\right)$. Solid lines: cracks and grains of similar size. Dotted lines: cracks much larger than the grains. A different color is used for each value of $\gamma$

assumed, no advanced percolation threshold develops when $\gamma \rightarrow 0$. In that case percolation occurs when $\eta \approx 1.158$ independently of $\gamma$. Accordingly, a homogenization approach where grains and cracks are treated separately, using e.g. Berryman's method for the polycrystal and an homogenization scheme for the cracks (e.g. MT, HS or SC), would give incorrect results, in the case where the cracks and grains have similar size and the grains have strong anisotropy. Such situation occurs in TATB polycrystals, as recently investigated [52].

To illustrate the predictions of $\mathrm{SC}^{\prime}$ when $G_{z p}=0$, the effective elastic moduli and thermal expansion coefficient are represented in Fig. (11) for various values of $E_{z}$, with $G_{z p}=0, E_{p}=1, \nu_{p}=\nu_{z p}=0.2$, $\alpha_{11}=1$ and $\alpha_{33}=0$. At $\eta=\eta_{c}^{\prime}$, method $\mathrm{SC}^{\prime}$ predicts $\widetilde{\nu} \approx 0.31$ independently of $E_{z}$ whereas $\widetilde{\alpha}=2 / 3$ as when $G_{z p} \neq 0$ (Fig. 6). 


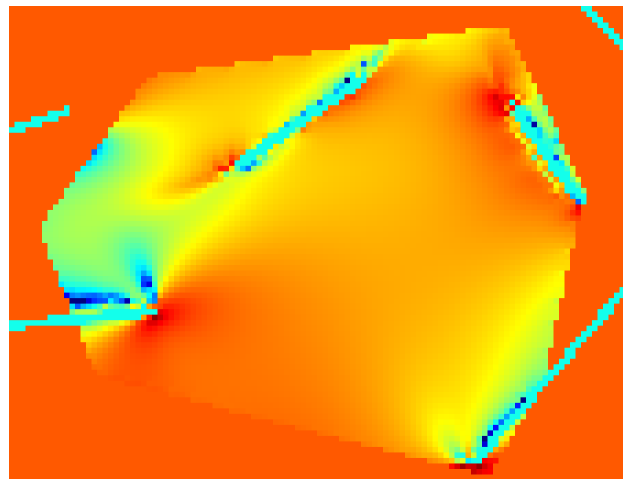

(a)

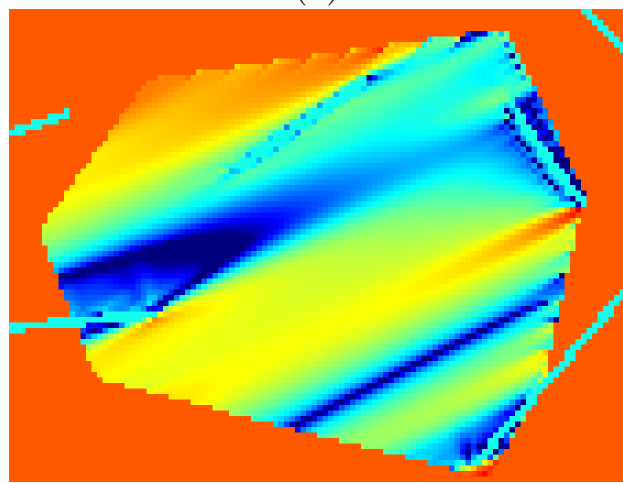

(c)

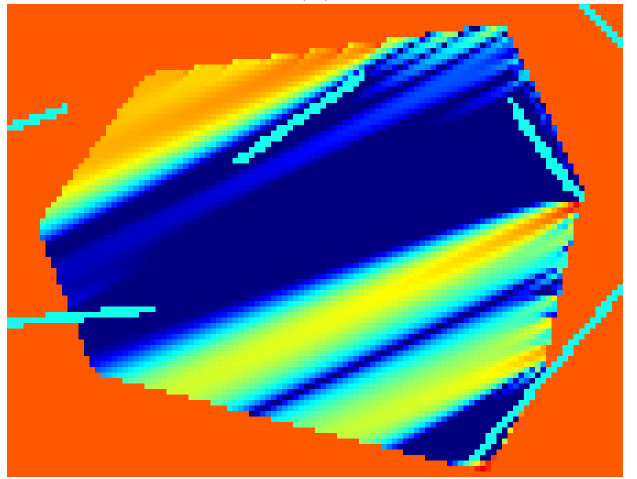

(e)

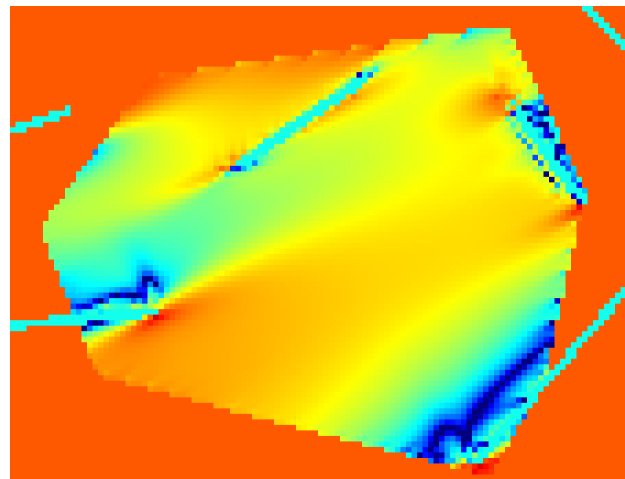

(b)

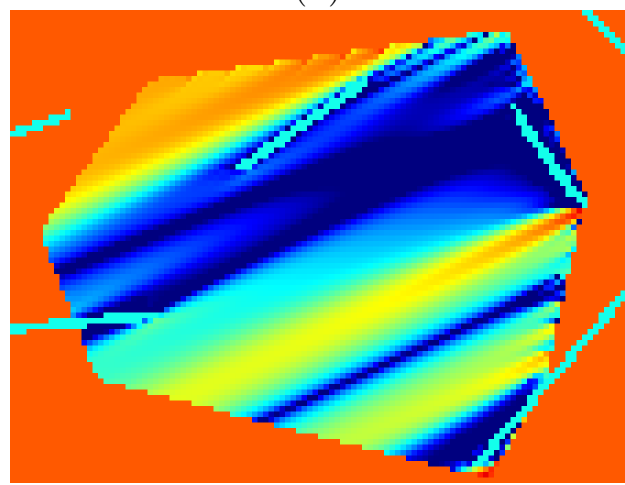

(d)

Figure 10. Map of the quantity $\log \left|\sigma_{33}\right|$ in a grain, thresholded in the range $[-22 ; 0.5]$ so that $\sigma_{33} \in\left[10^{-2} ; 3.16\right]$. The axis of symmetry $\mathrm{e}_{3}$ of the crystal is nearly-parallel to the plane of the $2 \mathrm{D}$ cut. Lowest values are indicated in blue, highest in red, and intermediate values in green-yellow. The same color scale is used for all maps (a-e). Cracks in light blue. The out-of-plane shear modulus is $G_{z p}=0.21$ (a), $0.04(\mathrm{~b}), 410^{-3}$ (c), $410^{-4}$ (d) and 0 (e). 


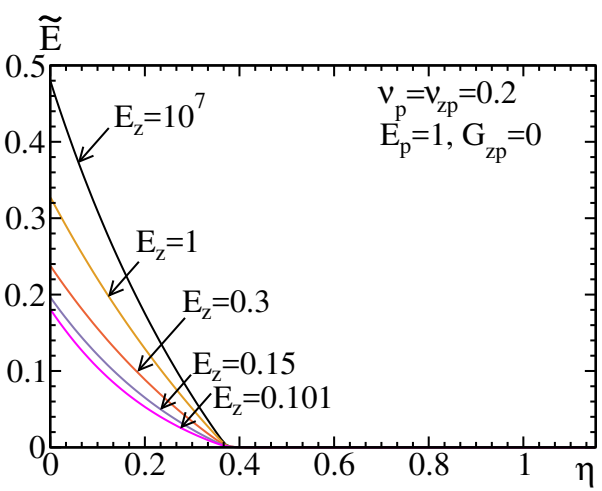

(a)

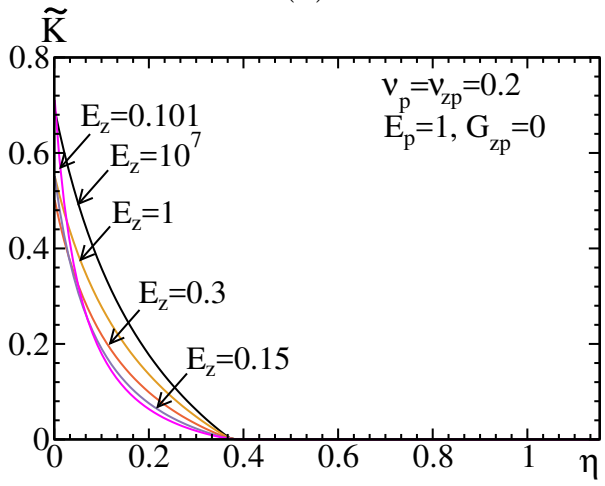

(c)

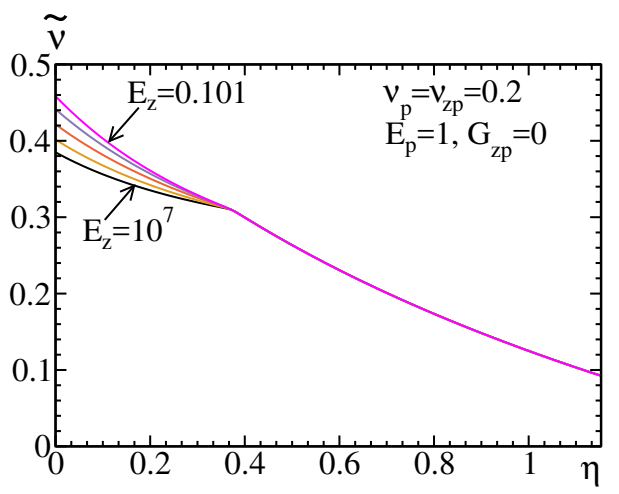

(b)

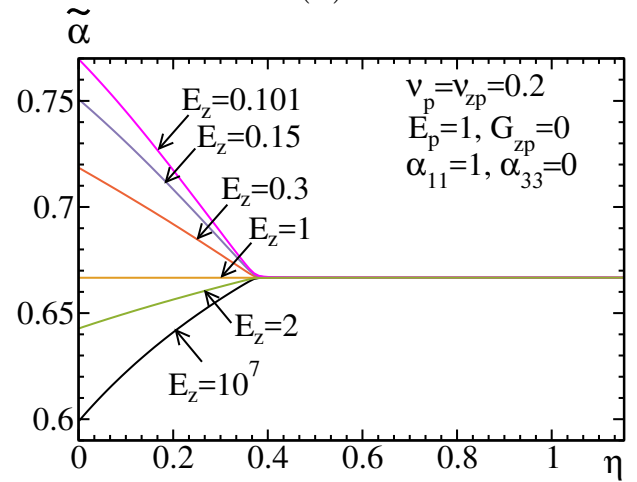

(d)

Figure 11. Effective response of a polycrystalline aggregate with out-of-plane shear modulus $G_{z p}=0$ as a function of the crack density parameter $\eta$, for various out-of-plane Young modulus $E_{z}$. (a) Young modulus $\widetilde{E}$, (b) Poisson coefficient $\widetilde{\nu}$, (c) bulk modulus $\widetilde{K}$, (d) thermal expansion coefficient $\widetilde{\alpha}=\widetilde{\alpha}_{V} / 3$.

\section{Conclusion}

In the present study, we have derived explicit formula that approximate the effective thermal and elastic, response of a polycrystal with hexagonal symmetry containing randomly-oriented cracks. This model is not an extension of that developed by Huang and $\mathrm{Hu}$ [21] for cubic symmetry. In particular, the coupling between crystal anisotropy and cracks is much stronger in the present case.

The main results are the following. First, in the absence of crystal anisotropy, i.e. for a homogeneous cracked body, our self-consistent approach yields estimates that are very close to that of a Boolean model of disk-shaped cracks. Good agreement is observed up to a crack-density parameter $\eta \approx 0.7$. In this domain, most cracks intersect another crack, and the matrix's Young modulus is weakened by as much as $75 \%$ for a Poisson ratio in the embedding medium of 0.2 . For general transversely-isotropic crystals, the self-consistent estimates predict a percolation threshold equal to about $\eta \approx 1.16$ for the crack density parameter.

Second, "advanced" percolation thresholds at $\eta=0.37$ and 0.75 are predicted for crystals with vanishing out-of-plane shear or Young modulus. The low percolation threshold predicted by the selfconsistent estimate in the former case can be related to the onset of "weakening" regions that surround the cracks and lie inside the grains. The existence of such regions has been confirmed by Fourierbased numerical computations carried out on a Voronoï-Boolean model. However, in contrast to the predictions of the self-consistent estimates, Fourier computations do not indicate a percolation threshold in highly-anisotropic crystals at low-crack density, but rather a strong weakening of the elastic moduli. The analytical estimates derived in the present approach fail to predict this mechanism.

Although the methodology presented herein has focused on cracked polycrystals, it is worth mentioning that such approach can be extended, in theory, to hexagonal polycrystals containing other types of inhomogeneities, including pores or rigid inclusions. 
Author contributions H.T. suggested the idea of research and formulated the model problem. D.J. computed the estimate for the geometrical percolation threshold. F.W. derived the self-consistent estimates and carried out the numerical computations. The manuscript was drafted by F.W. and subsequently extended and edited by D.J. and H.T. All the authors gave their final approval for publication.

Acknowledgements The financial support of the French Commissariat à l'Énergie Atomique is gratefuly acknowledged.

\section{References}

[1] F. Cormery and H. Welemane, A critical review of some damage models with unilateral effect., Mech. Res. Comm. 29 (2002), pp. 391-395.

[2] R. Bargellini, D. Halm, and A. Dragon, Modelling of quasi-brittle behaviour: a discrete approach coupling anisotropic damage growth and frictional sliding., Eur. J. Mech. A/Solids 27 (2008), pp. 564-581.

[3] F. Cormery and H. Welemane, A stress-based macroscopic approach for microcracks unilateral effect., Comput. Mat. Sci. 47 (2010), pp. 727-738.

[4] N. Challamel, A variationally based nonlocal damage model to predict diffuse microcracking evolution., Int. J. Mech. Sci. 52 (2010), pp. 1783-1800.

[5] B. Budiansky and R.J. O'Connell, Elastic moduli of a cracked solid, International Journal of Solids and Structures 12 (1976), pp. 81-97.

[6] H. Horii and S. Nemat-Nasser, Overall moduli of solids with microcracks: load-induced anisotropy, J. Mech. Phys. Solids 31 (1983), pp. 155-171.

[7] S. Andrieux, Y. Bamberger, and J. Marigo, Un modèle de matériau microfissuré pour les bétons et les roches, J. Méca. Théor. Appl. 5 (1986), pp. 471-513.

[8] D. Krajcinovic, Damage mechanics, Mech. Mat. 8 (1989), pp. 117-197.

[9] M. Kachanov, Effective elastic properties of cracked solids: critical review of some basic concepts, Applied Mechanics Reviews 45 (1992), pp. 304-335.

[10] V. Pensée and D. Kondo, Micromechanics of anisotropic brittle damage: comparative analysis between a stress based and a strain based formulation., Mech. Mat. 35 (2003), pp. 747-741.

[11] L. Dormieux and D. Kondo, Stress-based estimates and bounds of effective elastic properties: the case of cracked media with unilateral effects., Comput. Mat. Sci. 46 (2009), pp. 173-179.

[12] Q.Z. Zhu, J.F. Shao, and D. Kondo, A micromechanics-based thermodynamic formulation of isotropic damage with unilateral and friction effects., Eur. J. Mech. A/Solids 30 (2011), pp. 316-325.

[13] V. Monchiet, C. Gruescu, O. Cazacu, and D. Kondo, A micromechanical approach of crack-induced damage in orthotropic media: application to a brittle matrix composite, Engrg. Fract. Mech. 83 (2012), pp. 40-53.

[14] S. Levasseur, H. Welemane, and D. Kondo, A microcracks-induced damage model for initially anisotropic rocks accounting for microcracks closure., Int. J. Rock Mech. \& Mining Sci. 77 (2015), pp. 122-132.

[15] M. Ortiz, A constitutive theory for the inelastic behavior of concrete, Mechanics of materials 4 (1985), pp. 67-93.

[16] G. Pijaudier-Cabot and B.Z. P., Nonlocal damage theory., J. Engrg. Mech. 113 (1987), pp. 1512-1533.

[17] R.G. Naum and C.K. Jun, Thermal expansion of polycrystalline graphite., Journal of Applied Physics 41 (1970), pp. 5092-5095.

[18] L. Delannay, P. Yan, J.F.B. Payne, and N. Tzelepi, Predictions of inter-granular cracking and dimensional changes or irradiated polycrystalline graphite under plane strain, Computational Materials Science 87 (2014), pp. 129-137.

[19] J.R. Kolb and H.F. Rizzo, Growth of 1,3,5-Triamino-2,4,6-Trinitrobenzene (TATB). I. anisotropic thermal expansion., Propellants, Explosives, Pyrotechnics 4 (1979), pp. 10-16.

[20] A. Ambos, F. Willot, D. Jeulin, and H. Trumel, Numerical modeling of the thermal expansion of an energetic material, International Journal of Solids and Structures 60-61 (2015), pp. 125-139.

[21] Y. Huang and K.X. Hu, Elastic moduli of a microcracked composite with spherical inclusions of cubic anisotropy, Composites science and technology 50 (1994), pp. 149-156.

[22] N. Laws, A note on penny-shaped cracks in transversely isotropic materials, Mechanics of Materials 4 (1985), pp. 209-212.

[23] C. Gruescu, V. Monchiet, and D. Kondo, Eshelby tensor for a crack in an orthotropic elastic medium, Comptes Rendus Mecanique 333 (2005), pp. 467-473.

[24] J.R. Bristow, Microcracks, and the static and dynamic elastic constants of annealed and heavily cold-worked metals, British Journal of Applied Physics 11 (1960), p. 81.

[25] R. Hill, A self-consistent mechanics of composite materials, Journal of the Mechanics and Physics of Solids 
13 (1965), pp. 213-222.

[26] I. Sevostianov, N. Yilmaz, V. Kushch, and V. Levin, Effective elastic properties of matrix composites with transversely-isotropic phases, International Journal of Solids and Structures 42 (2005), pp. 455-476.

[27] T. Mura, Micromechanics of defects in solids, Martinus Nijhoff Publishers, The Hague, 1982.

[28] V.M. Levin, M.I. Rakovskaja, and W.S. Kreher, The effective thermoelectroelastic properties of microinhomogeneous materials, International Journal of Solids and Structures 36 (1999), pp. 2683-2705.

[29] I. Sevostianov and M. Kachanov, Explicit cross-property correlations for anisotropic two-phase composite materials, Journal of the Mechanics and Physics of Solids 50 (2002), pp. 253-282.

[30] Wolfram Research, Inc., Mathematica software version 10.2 (2015). Champaign, Illinois.

[31] Y. Benveniste, Universal relations in piezoelectric composites with eigenstress and polarization fields, Part 2: Multiphase media-effective behavior, Journal of Applied Mechanics 60 (1993), pp. 270-274.

[32] J.G. Berryman, Bounds and self-consistent estimates for elastic constants of random polycrystals with hexagonal, trigonal, and tetragonal symmetries, Journal of the Mechanics and Physics of Solids 53 (2005), pp. 2141-2173.

[33] Z. Hashin, Thermal expansion of polycrystalline aggregates: I. Exact analysis, Journal of the Mechanics and Physics of Solids 32 (1984), pp. 149-157.

[34] Z. Hashin, Analysis of composite materials-a survey, Journal of Applied Mechanics 50 (1983), pp. 481-505.

[35] G.W. Milton, The Theory of Composites, Cambridge Univ. Press, Cambridge, 2002.

[36] M. Avellaneda, Iterated homogenization, differential effective medium theory and applications, Communications on Pure and Applied Mathematics 40 (1987), pp. 527-554.

[37] E. Kröner, Bounds for effective elastic moduli of disordered materials, Journal of the Mechanics and Physics of Solids 25 (1977), pp. 137-155.

[38] J.G. Berryman, Long-wavelength propagation in composite elastic media ii. ellipsoidal inclusions, The Journal of the Acoustical Society of America 68 (1980), pp. 1820-1831.

[39] P.N. Sævik, M. Jakobsen, M. Lien, and I. Berre, Anisotropic effective conductivity in fractured rocks by explicit effective medium methods, Geophysical Prospecting 62 (2014), pp. 1297-1314.

[40] P.N. Sævik, I. Berre, M. Jakobsen, and M. Lien, A 3d computational study of effective medium methods applied to fractured media, Transport in porous media 100 (2013), pp. 115-142.

[41] E.H. Saenger, O.S. Krüger, and S.A. Shapiro, Effective elastic properties of randomly fractured soils: 3d numerical experiments, Geophysical Prospecting 52 (2004), pp. 183-195.

[42] R. Rosenzweig, V.V. Mourzenko, J.F. Thovert, and P.M. Adler, Solid matrix partition by fracture networks, Physical Review E 90 (2014), p. 022407.

[43] Y.B. Yi and K. Esmail, Computational measurement of void percolation thresholds of oblate particles and thin plate composites, Journal of Applied Physics 111 (2012), p. 124903.

[44] T. Bretheau and D. Jeulin, Caractéristiques morphologiques des constituants et comportement à la limite élastique d'un matériau biphasé fe/ag, Revue de Physique Appliquée 24 (1989), pp. 861-869.

[45] Y.B. Yi and E. Tawerghi, Geometric percolation thresholds of interpenetrating plates in three-dimensional space, Physical Review E 79 (2009), p. 041134.

[46] Y. Benveniste, A new approach to the application of Mori-Tanaka's theory in composite materials, Mechanics of materials 6 (1987), pp. 147-157.

[47] P. Ponte Castañeda and J.R. Willis, The effect of spatial distribution on the effective behavior of composite materials and cracked media, Journal of the Mechanics and Physics of Solids 43 (1995), pp. 1919-1951.

[48] F. Willot and Y.P. Pellegrini, Fast Fourier Transform computations and build-up of plastic deformation in 2D, elastic-perfectly plastic, pixelwise-disordered porous media, in D. Jeulin, S. Forest (eds), "Continuum Models and Discrete Systems CMDS 11", Paris. École des Mines, 2008, pp. 443-449, online at https: //arxiv.org/abs/0802.2488.

[49] F. Willot, Fourier-based schemes for computing the mechanical response of composites with accurate local fields, Comptes Rendus Mécanique 343 (2015), pp. 232-245.

[50] J.B. Gasnier, F. Willot, H. Trumel, D. Jeulin, and J. Besson, Thermoelastic properties of microcracked polycrystals. Part I: Adequacy of Fourier-based methods for cracked elastic bodies, International Journal of Solids and Structures 155 (2018), pp. 248-256.

[51] F. Willot, Y.P. Pellegrini, M. Idiart, and P. Ponte Castañeda, Effective-medium theory for infinite-contrast two-dimensionally periodic linear composites with strongly anisotropic matrix behavior: dilute limit and crossover behavior, Physical review B 78 (2008), p. 104111.

[52] J.B. Gasnier, F. Willot, H. Trumel, D. Jeulin, and M. Bessy, Thermoelastic properties of microcracked polycrystals. Part II: The case of jointed polycrystalline TATB, International Journal of Solids and Structures 155 (2018), pp. 257-274. 